\title{
ATRIBUTOS DE SOLO NA ESTIMATIVA DA PRODUTIVIDADE DE CAFÉ ARÁBICA ATRAVÉS DE REGRESSÃO MÚLTIPLA
}

\author{
Jorge Tadeu Fim Rosas ${ }^{1}$ \\ Danielle Inácio Alves ${ }^{2}$ \\ Michel de Assis Silva ${ }^{3}$ \\ Julião Soares de Souza Lima ${ }^{4}$ \\ Samuel de Assis Silva ${ }^{5}$
}

Resumo: Objetivou-se, neste estudo, através de regressão múltipla, estimar a produtividade do café arábica, a partir dos atributos do solo. A pesquisa foi desenvolvida em uma área comercial de aproximadamente 1,2 ha, cultivada com Coffea arabica L., variedade Catucaí Amarelo 20/15 - 479. Para a realização das amostragens de solo e da produtividade foi montada uma uma malha de 100 pontos. As informações geográficas dos pontos foram obtidas com auxílio de estação total. Aos dados foram ajustados modelos de regressão linear múltipla para estimar essa variável em função dos atributos de solo. A análise geoestatística foi realizada para os dados reais de produtividade e para a estimada por regressão múltipla. O modelo que melhor se ajustou à distribuição múltipla dos dados foi o linear, sendo capaz de descrever a relação entre a produtividade e os atributos originais de solo. Na análise geoestatística, todas as variáveis apresentaram variogramas com patamares bem definidos. A produtividade estimada por regressão múltipla assemelhou-se à produtividade real, indicando que o modelo ajustado foi capaz de descrever com eficiência a sua variação.

Palavras-chave: Agricultura de precisão; Cafeicultura; Estatística multivariada.

\footnotetext{
1 Departamento de Engenharia Rural/Universidade Federal do Espírito Santo, Brasil. E-mail: jorgetadeufimrosas@hotmail.com.

2 Departamento de Engenharia Rural/Universidade Federal do Espírito Santo/Universidade Federal do Espírito Santo, Brasil. E-mail: danielle.inacio@hotmail.com.

3 Departamento de Engenharia Rural Universidade Federal do Espírito Santo, Brasil. E-mail: michelsilv@outlook.com.

4 Departamento de Engenharia Rural/Universidade Federal do Espírito Santo, Brasil. E-mail: limajss@yahoo.com.br.

5 Departamento de Engenharia Rural/ Universidade Federal do Espírito Santo, Brasil. E-mail: sasilva@pq.cnpq.br.
} 